\title{
The common occurrence of errors of perceived distance
}

\author{
WALTER C. GOGEL \\ University of California, Santa Barbara, California 93106
}

\begin{abstract}
The effect on the perceived distance of a test object of fixating to a distance different from that of the test object was investigated using monocular observation and two methods for measuring perceived distance. One method, the size adjustment procedure, applying the size-distance invariance hypothesis, measured perceived distance by measuring perceived size. The results from this method were compared with those from a head-motion procedure which used the apparent concomitant motion resulting from head motion to measure perceived distance. The results from both procedures indicated that the apparent distance of the test object physically located at a constant distance varied directly as a function of the fixation distance. This occurred despite the presence of texture on the walls and floor of the visual alley. These and other perceptual effects are interpreted as demonstrating that errors in perceived distance (contrary to the theory of direct perception) are a common occurrence in ordinary visual fields.
\end{abstract}

It has been found that test objects tend to be displaced perceptually toward the perceived distance of a fixated object (Wist \& Summons, 1976). It has also been found that an error in the perception of the distance of a physically stationary object will result in the object appearing to the observer to move horizontally with a horizontal movement of the head (Gogel \& Tietz, 1973). This illusory motion, concomitant with the motion of the head, will be in the same direction as the head motion, e.g., the object will appear to move left as the head moves left, or opposite to the head motion, e.g., the object will appear to move right as the head moves left, depending upon whether the object appears to be nearer or more distant than its physical distance, respectively. Taken together, these two findings suggest that the apparent concomitant motion and hence the apparent distance of a physically stationary, nonfixated test object will vary systematically with the perceived distance of the object on which the observer is fixated. This prediction was tested and confirmed in a recent study by Gogel and Tietz (1977). Also, it was noted that, under usual as well as under laboratory conditions, fixating an object while moving the head often will produce illusory concomitant motions for objects at distances other than the fixation distance. This can be readily demonstrated, for example, by fixating a portion of a window frame while moving the head and noting that objects viewed through the window appear to move in the same

This research was supported by Research Grant BNS 77-16620 from the National Science Foundation. The author wishes to thank Jerome D. Tietz for his assistance in constructing the apparatus and analyzing the data of the experiment and Kenneth Pulliam for collecting the data. direction as the head motion. It was concluded from this that errors in perceived distance occur often in portions of the environment to which our gaze is not momentarily directed.

The notion that the visual system is prone to errors in perceived distance, particularly the perceived distance of nonfixated objects, is of considerable theoretical significance. For example, it is opposed to the view that errors in the perception of distance are restricted to reduced conditions of observation (Gibson, 1966), and it suggests that, if veridical perceptions of distance are achieved throughout an extended visual field, some synthesis of distance information from successive fixations is likely to have occurred.

An illusory object motion concomitant with head motion is not the only perceptual change that results from an error in perceived distance. Another is a change in the apparent size of the object or, more generally, a change in the apparent linear extent per unit of linear extent on the eye. For example, for a constant size on the eye, perceived size, as measured in a frontoparallel plane, will vary directly with the perceived distance of the plane. This phenomenon is a special case of the size-distance invariance hypothesis known as Emmert's law (see Kilpatrick \& Ittelson, 1953). The present study examines further the hypothesis that the perceived distance of a test object is modified as a function of the distance of fixation. As will be discussed below, the method used is to measure the perceived distance of the test object applying both the size-distance invariance hypothesis (the size adjustment procedure) and apparent concomitant motion (the head-motion procedure). If both of these procedures reflect the expected change 
in perceived distance, this will support the conclusion that the frequent occurrence of apparent concomitant motions of nonfixated objects in normal situations indicates that errors in the perceived distances of these objects are equally frequent.

\section{Measuring Perceived Distance by the Size Adjustment Procedure}

Figure 1 is a top-view schematic drawing illustrating the size adjustment procedure for measuring perceived distance used in the present study. With this procedure the head is stationary. This figure illustrates the situation in which a point of light, physically at a distance $D_{m}$, moves repetitively right and left with the right-left extent of this physical horizontal motion (S) adjustable by the observer. The task of the observer is to adjust the physical extent of horizontal motion $\left(S_{n}\right.$ or $S_{f}$ in the figure) until the perceived extent $S_{n}^{\prime}$ or $S_{f}^{\prime}$ is a designated amount (e.g., either 3 or 5 in.). Suppose that, under one condition, the point appears at the distance $D_{n}^{\prime}$ and, under another condition at another time, at $D_{f}^{\prime}$. According to the size-distance invariance hypothesis,

$$
\mathbf{D}^{\prime}=\mathrm{CDS}^{\prime} / \mathrm{S} \text {, }
$$

where $\mathbf{C}$ is an observer constant and the physical distance $\mathrm{D}$ of the point is constant in this study at $D_{m}$. It follows, from Equation 1 and Figure 1, that the $S$ at $D_{m}$ adjusted to produce a constant perceived extent of motion $\left(S_{n}^{\prime}=S_{f}^{\prime}\right)$ will be less if the perceived distance of the point is $D_{f}^{\prime}$ rather than $D_{n}^{\prime}$. Conversely, if the physical extent of motion, $S$, of the point at a constant $\mathrm{D}$ that is required in order to produce a constant perceived extent of motion $S^{\prime}$ is less under one condition than another, the perceived distance of the point must be greater under the former condition. Using Equation 1, the perceived distance, $D_{n}^{\prime}$ or $D_{f}$, of the point of light, as a function of the distance of fixation in the experiment, can be computed from the value of $S_{n}$ or $S_{f}$ adjusted by the observer in order to achieve the given perceived extent $S^{\prime}=3$ or 5 in. For the purposes of this study, $\mathrm{C}$ will be considered to be unity. If the apparent distance, $D^{\prime}$, of the point of light calculated from Equation 1 changes in the predicted direction as a function of fixation distance, the conclusion that systematic errors in the perceived distance of a nonfixated object can occur as a function of the distance of fixation will be supported.

\section{Measuring Perceived Distance by the Head Motion Procedure}

The apparent concomitant motion resulting from an error in perceived distance has been used in several studies to measure apparent distance (Gogel, 1976, 1977; Gogel \& Tietz, 1977) and is called the head-

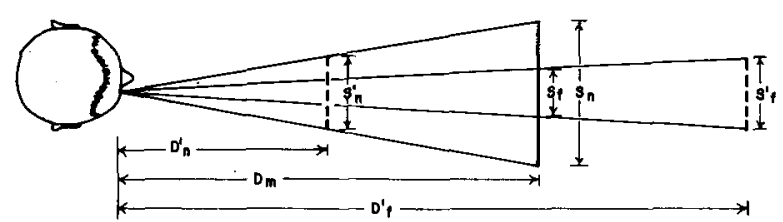

Figure 1. The physical extent, $S_{n}$ or $S_{f}$ of horizontal motion of a point of light physically at $D_{m}$ required in order to perceive a constant extent of motion $\left(S_{n}^{\prime}=S_{\mathbf{f}}\right.$ ) as a function of perceiving the point at $D_{n}^{\prime}$ or $D_{f}^{\prime}$.

motion procedure. In one variation of this procedure the observer moves his head horizontally and adjusts the path of motion of a point of light to be perceptually vertical. If, instead of being physically stationary, the point of light (the test object) is physically moved vertically as the head is moved horizontally, two components of perceived motion concomitant with the head motion are present simultaneously. Since one of these, the illusory component, is horizontal and the other, the perception of the real motion, is vertical, the point will appear to move at a slant concomitant with the head motion. The angle of the perceived path of motion of the point will be determined by the ratio of these two components. The larger the illusory component relative to the apparent vertical component, the more nearly horizontal will be the resulting path of perceived motion of the point as the head is moved horizontally.

The relation between the perceived slant $\alpha^{\prime}$ of the path of motion of the point and the perceived distance of the point for a physical vertical motion, $V$, of the point is illustrated in $\mathbf{A}$ of Figures 2 and 3. Figure $2 \mathrm{~A}$ represents the situation in which a point of light at a distance $D_{m}$ from the observer physically moves upward (from e to $f$ ) as the head is moved from Position 1 to Position 2, and downward (from $f$ to $e$ ) as the head is moved from Position 2 to Position 1. The physical distance of the point of light in this case is the distance of the pivot around which the direction between the observer and the point rotates as the head is moved and is called the pivot distance, $D_{p}$. Suppose that the point of light is perceived at a closer distance $\left(D_{n}^{\prime}\right)$ than its pivot distance. As indicated in Figure $2 \mathrm{~A}$, this will produce an illusory component of apparent concomitant motion, $W^{\prime}$, that will be in the same direction as the head motion. A component of perceived motion, $V^{\prime}$, resulting from the physical motion of the point also will be present. As shown in Figure 2A, the simultaneous occurrence of $\mathrm{V}^{\prime}$ and $\mathrm{W}^{\prime}$ will result in the point of light appearing to move between lower left and upper right at an apparent slant $\alpha^{\prime}$. Figure 3A indicates the perceived slant of the point again moving vertically between $e$ and $f$ as the head moves between Positions 1 and 2, but in this case, the apparent distance of the point $\left(D_{f}^{\prime}\right)$ is greater than its 


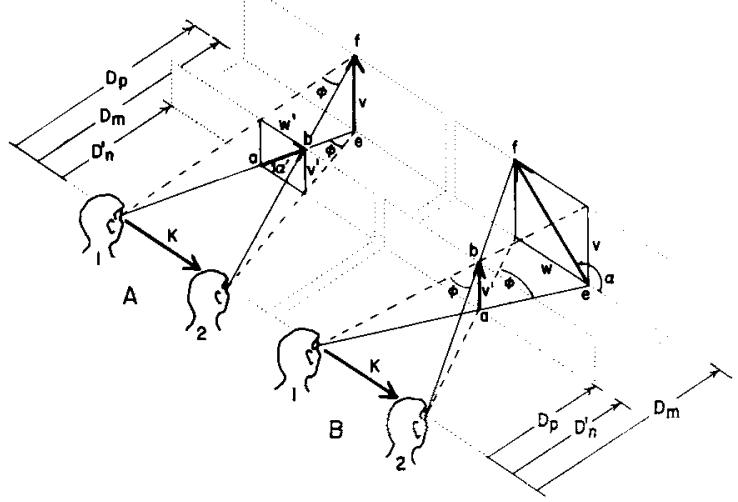

Figure 2. A point of light physically at $D_{m}$ and perceptually at the closer distance $D_{n}^{\prime}$ moves up and down concomitant with the right and left motion, respectively, of the head. Figure $2 \mathrm{~A}$ illustrates the slant of the apparent path of motion $\left(\alpha^{\prime}\right)$ of the point for the situation in which the point appears closer to the observer than the pivot distance $\left(D_{p}\right)$, where $D_{p}=D_{m}$. Figure $2 B$ illustrates the slant of the physical path of motion $(\alpha)$ of the point required in order for the point perceptually at $D_{n}^{\prime}$ to appear to move vertically, where $D_{p}=D_{n}^{\prime}$.

pivot distance. As diagramed, the point will appear to move between lower right and upper left concomitant with the horizontal motion of the head.

The pivot distance, $D_{p}$, in both Figures $2 A$ and $3 A$ is at the physical distance, $\mathrm{D}_{\mathrm{m}}$, of the point of light. As shown in Figures $2 \mathrm{~B}$ and $3 \mathrm{~B}$, by changing the pivot distance while keeping the physical distance constant, the apparent orientation of the path of concomitant motion of the point can be adjusted until the point of light appears to be moving vertically $\left(\alpha^{\prime}=90^{\circ}\right.$ and $\left.\mathrm{W}^{\prime}=0\right)$. This is accomplished by adjusting the physical slant of motion of the point of light at $D_{m}$ until $D_{p}=D_{n}^{\prime}$, in the case of Figure 2B, and until $D_{p}=D_{f}^{\prime}$, in the case of Figure 3B. The physical slant $(\alpha)$ required to achieve $\alpha^{\prime}=90^{\circ}$, if the perceived distance of the point is at $D_{n}^{\prime}$ or $D_{f}^{\prime}$, is shown in Figures 2B and 3B, respectively. For the phase of head motion and vertical point motion shown in Figures $2 \mathrm{~A}$ and $3 \mathrm{~A}$, this physical slant is counterclockwise from the vertical if $D^{\prime}<D$ (Figure 2B) and clockwise from the vertical if $\mathrm{D}^{\prime}>\mathrm{D}$ (Figure 3B). According to the geometry of Figures $2 B$ and $3 B$, the perceived distance, $D^{\prime}$, of the point can be calculated from the physical concomitant motion, $\mathrm{W}$, required in order for the point of light to appear to move vertically. The equation for this calculation (Gogel \& Tietz, 1977) is

$$
\mathrm{D}^{\prime}=\mathrm{KD} /(\mathrm{K}-\mathrm{W})
$$

where $\mathrm{W}$ is taken as positive when the physical concomitant motion is in the same direction as the head motion and as negative when the physical concomitant motion is opposite to the direction of the head motion. It is hypothesized, consistent with the study by Gogel and Tietz (1977) and with the results expected from the size adjustment procedure, that the $\mathrm{D}^{\prime}$ of the test point, calculated from $\mathrm{W}$ and Equation 2, will be less for the near than for the far distance of fixation.

It is not hypothesized that the $\mathrm{D}^{\prime}$ of the test point necessarily will be different from $D$ at both the near and far fixation distance. The reason for this is that superposed upon the effect of fixation distance is an effect on the perceived distance of the test point attributable to another factor called the specific distance tendency (Gogel \& Tietz, 1973). This is the tendency in the absence of effective distance cues for the observer to perceiv. the test object at 2 or $3 \mathrm{~m}$. If the test point is closer than this to the observer, the specific distance tendency will add to the error in the $\mathrm{D}^{\prime}$ of the test point resulting from the far fixation and subtract from the error in the $\mathrm{D}^{\prime}$ of the test point resulting from the near fixation. For example, it was found in Experiment II in the study by Gogel and Tietz (1977), that only when the physical distance of the test point (previously at $135 \mathrm{~cm}$ ) was increased to about $2 \mathrm{~m}$ was the fixation to a closer distance able to modify the $\mathrm{D}^{\prime}$ of the test point to be substantially less than its physical distance. Since a relatively close distance $(97.5 \mathrm{~cm})$ is used with the test point in the present study, it is likely that the specific distance tendency will decrease the error in $\mathrm{D}^{\prime}$ of the test point expected from the near fixation distance but will increase it at the far fixation distance. The effect of the specific distance tendency on the perceived distance of the test point can be evaluated by

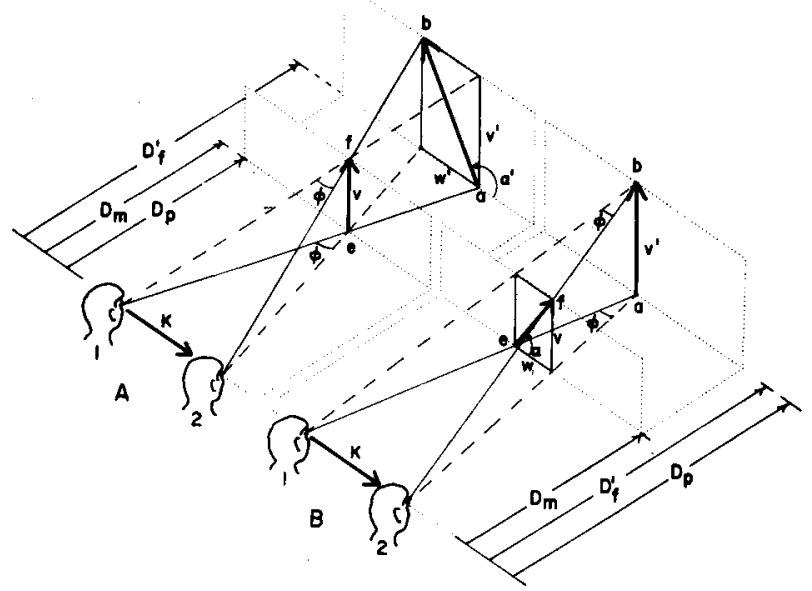

Figure 3. A point of light physically at $D_{m}$ and perceptually at the farther distance $D_{f}^{\prime}$ moves up and down concomitant with the right and left motion, respectively, of the head. Figure $3 \mathrm{~A}$ illustrates the slant of the apparent path of motion $\left(\alpha^{\prime}\right)$ of the point for the condition in which the point appears more distant from the observer than the pivot distance $\left(D_{p}\right)$, where $D_{p}=D_{m}$. Figure 3B illustrates the slant of the physical path of motion $(\alpha)$ of the point required in order for the point perceptually at $D_{f}^{\prime}$ to appear to move vertically, where $D_{p}=D_{f}^{\prime}$. 
including a condition in which the fixation is on the test object as well as to distances nearer and farther than the test object. Regardless of the effect of the specific distance tendency, however, the computed value of $D^{\prime}$ of the point should be less with the near than with the far distance of fixation.

\section{METHOD}

\section{Apparatus}

One requirement of the experiment was that a test point of light perceptually suspended in the visual field be capable of being moved concomitant with the horizontal motion of the head along a path slanted at an angle that could be varied, preferably by the observer. Another requirement was that, with the head stationary, the horizontal extent of the motion of the test point could be varied, again preferably by the observer. These requirements were met by an apparatus that allowed the path of motion of a point of light on a display module to be changed continuously. A third requirement was that the fixation objects be perceived as being at distances closer and more distant than the physical distance of the test point so that the expected modification of the perceived distance of the test point would be substantial. A convenient way of accomplishing the last requirement was to have the fixation objects standing on a patterned alley floor so as to appear at the near and far portions of the alley with the test point physically located between these two distances.

A schematic diagram of the apparatus used in the experiment is shown in Figure 4. The observer sat on a stool in a lightproof booth and monocularly viewed an alley, $50 \mathrm{~cm}$ wide and extending optically $194 \mathrm{~cm}$ from himself, through an aperture, $30 \mathrm{~cm}$ wide and $15 \mathrm{~cm}$ high, with the center of the aperture at eye level. Closing a shutter at the aperture occluded the view of the alley. The floor of the alley was $15 \mathrm{~cm}$ below eye level, with the floor and two sides of the alley covered with a black cloth containing white polka dots, $2.3 \mathrm{~cm}$ in diameter and separated by $2.5 \mathrm{~cm}$. The back wall of the alley was covered with black cloth. A Conrac CRT display module for presenting the moving point of light was located in the alley at $97.5 \mathrm{~cm}$ from the observer, with the face of the display tube perpendicular to the alley and in a frontoparallel plane of the observer. A partially reflecting, partially transmitting

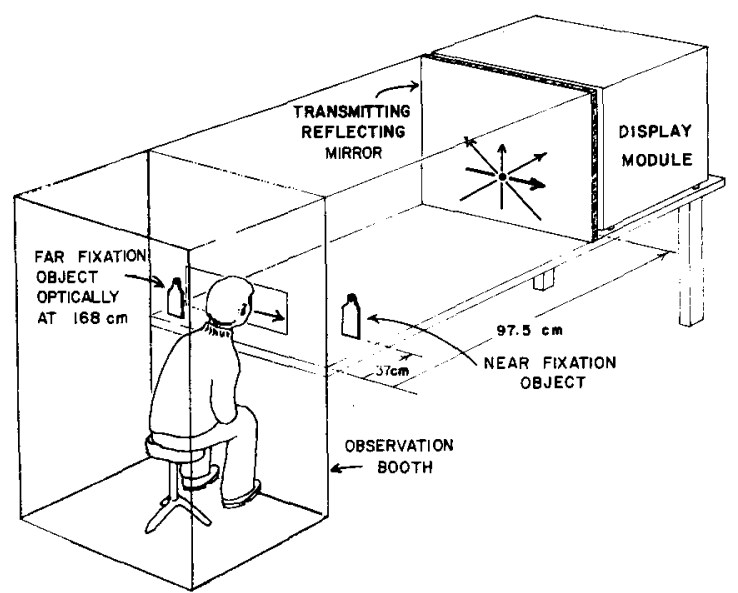

Figure 4. A schematic drawing of the observation booth, visual alley, and several possible motions of the point of light on the display module. The floor and side walls of the visual alley were covered with textured cloth and were optically extended by reflection from a reflecting-transmitting mirror located directly in front of the motion display. mirror placed $.4 \mathrm{~cm}$ in front of the face of the display module optically extended the length of the alley by reflection when viewed from the observation position. The alley was illuminated from above by a white incandescent lamp. In order to eliminate the visibility of the aftertrace of the moving point on the display module, a red acetate sheet, invisible to the observer as a surface, covered the viewing aperture. The red filter made the test point appear red and gave the impression that the visual alley was illuminated by red light. The luminous intensity of the moving point was $1.5 \times 10^{-5} \mathrm{~cd}$, as measured from the observation position with a spectra brightness spot meter.

The two fixation objects were upright white figures standing on the alley floor. These were curved at the top (see Figure 4). A black spot, $1.3 \mathrm{~cm}$ in diameter and located at eye level near the top of each white figure, was the portion of the fixation object to which the observer directed his gaze. The near fixation object was $15.3 \mathrm{~cm}$ from the right edge of the visual alley and $37 \mathrm{~cm}$ from the observer. The back of this object was black so that it could be seen by the observer only directly and not by reflection from the mirror. The white surface (and black spot) of the far fixation object faced the mirror, not the observer. This fixation object, located $2 \mathrm{~cm}$ from the left edge of the alley, was not visible directly to the observer and could only be seen by reflection from the mirror. The optical distance of the far fixation point (observer to mirror to fixation object) was $168 \mathrm{~cm}$. The right-left direction of the near and far fixation objects were not reversed in the experiment since, if the near fixation object had been positioned on the left, the test point would sometimes have disappeared on the blind spot of the eye as the head was moved when using the head-motion procedure. Upon looking into the visual alley, the observer saw the pattern-covered floor and walls, a near and a far fixation object, and a luminous red point of light suspended in the alley and viewed against the dark back wall of the alley. Neither the mirror nor the surface of the display module was visible to the observer at any time during the experiment. The alley was visually indistinguishable from a textured alley extending physically to $194 \mathrm{~cm}$ and containing a point of light without any visible support moving in a frontoparallel plane at a distance of $97.5 \mathrm{~cm}$ with two fixation objects, one at $37 \mathrm{~cm}$ and the other at $168 \mathrm{~cm}$, standing on the alley floor.

Head-motion apparatus. A head- and chinrest at the observation position was mounted on ball bearings and was movable left and right by the observer through a distance of $17.5 \pm .25 \mathrm{~cm}$. The lateral motion of the head- and chinrest was limited by cushioned stops at each end of its travel. For the head-motion procedure, the observer moved his head so as to arrive at the left and right stops in synchrony with clicks from a metronome presented over a loudspeaker at a rate of one every $1.5 \mathrm{sec}$. To assist in moving his head in the head- and chinrest, with his left hand the observer grasped a support bar attached to the head- and chinrest. The horizontal motion of the head varied the resistance of two linear potentiometers connected to the head- and chinrest by means of a rack-and-pinion gear. The potentiometers, in turn, modulated two voltage sources with the voltages from these sources applied to the display module to control the horizontal and vertical components of motion of the test point of light. The magnitude of the physical horizontal component of motion (W of Figures 2B and $3 \mathrm{~B}$ ) on the display module per centimeter of horizontal displacement of the head could be adjusted by the observer by turning a knob located near the observation position, until the point of light appeared to move vertically as the head was moved left and right in the head-and chinrest. Turning the knob varied the physical slant of the concomitant motion of the point of light by changing the ratio of its horizontal-to-vertical motion with the vertical component always $12.3 \mathrm{~cm}\left(7.2^{\circ}\right.$ of visual angle) for the $17.5-\mathrm{cm}$ motion of the head. The direction of the physical horizontal motion of the point of light, with or against the head motion, was controlled by the experimenter using a switch located outside the observation booth. The physical extent of horizontal 
motion of the point could be determined by the experimenter from the reading on a digital voltmeter. From this value of $W$, the experimenter calculated the perceived distance, $D^{\prime}$, of the point, using Equation 2.

Size adjustment apparatus. For the measurement of perceived distance using the size adjustment procedure, the head- and chinrest was pinned in a position so that the right eye of the observer was centered at the viewing aperture. With the head- and chinrest fixed in this position, the point of light was made to move horizontally through an extent determined by the knob adjustment of the observer. This was accomplished by using another rack-andpinion gear assembly that was not connected to the head-and chinrest but, instead, was moved horizontally through $17.5 \mathrm{~cm}$ by the experimenter at the same rate as that used for the head motion. The magnitude of the right-left motion produced on the display module as the experimenter moved this assembly back and forth could be varied by the observer by turning the same knob used with the head-motion procedure. The observer adjusted this knob until the right-to-left extent of the motion of the test point appeared to him to be the size specified by the instructions (either 3 or 5 in.). Again, the voltage modulated by the linear potentiometer attached to the adjustment knob of the observer was converted by the experimenter to centimeters of physical horizontal motion of the point $\left(S_{n}\right.$ or $S_{f}$ of Figure 1) by means of readings taken from the digital voltmeter. From the values of $S$, the $D^{\prime}$ of the test point was calculated using Equation 1 and assuming that $\mathrm{C}=1$.

\section{Observers}

The observers were 48 students enrolled in an introductory course in psychology who, by their participation in the experiment, satisfied a course requirement. All had at least 20/20 acuity in their right eye, both near and far (without glasses or contact lenses), as measured on the Keystone Orthoscope, and were naive as to the purpose of the experiment.

\section{Procedure}

Monocular observation (right eye only) was used throughout this study. The observers had three primary tasks. One was to adjust the path of the test point to be apparently vertical while moving the head. Another was to adjust the extent of the horizontal motion of the test point (with stationary head) to be either 3 or 5 in. Another task was to verbally report the apparent distance of the test point in feet or inches or in some combination of both. Each of these tasks was completed (1) while fixating the fixation spot at the near $(\mathrm{N})$ distance, (2) while fixating the moving test point at the middle (M) distance, and (3) while fixating the fixation spot at the far (F) distance. Two other tasks which were performed less frequently were to indicate verbally the apparent distance of the near and of the far fixation spot in feet or inches or some combination of both, and to adjust the lateral separation between two metal posts located at waist level in the observation booth to either 3 or 5 in. (with the observation shutter closed). Half of the observers used the 3-in. criterion, and half used the 5 -in. criterion. For all observers, either the head-motion procedure or the size adjustment procedure for measuring apparent distance was used at all three fixation distances (N, M, or F) before obtaining the measures with the remaining procedure at these fixation distances. Half of the observers used the head-motion procedure before the size adjustment procedure; the order was reversed for the remaining observers.

A verbal report of the apparent distance of the test point followed each adjustment of physical slant to the apparent vertical and each adjustment of apparent horizontal size. Upon completion of the slant adjustments and verbal reports or the size adjustments and verbal reports at the three fixation distances, either verbal reports of the apparent distance of the near and far fixation objects or post adjustments in the observation booth to indicate either 3 or 5 in. were obtained. The order in which the near, middle, and far fixation distance was used was the same for a particular observer with both the head-motion and size adjustment procedures, with these orders counterbalanced between observers.

\section{RESULTS}

The values of $\mathrm{S}$ and $\mathrm{W}$ obtained from the size adjustments and head-motion procedures, respectively, and the values of $D^{\prime}$ found by substituting $S$ and W in Equations 1 and 2, respectively, are summarized in Table 1. The hypothesis to be tested is that the size adjustment procedure, in addition to the headmotion procedure, resulted in a measured increase in the perceived distance $\left(D^{\prime}\right)$ of the point of light as a function of the increase in the distance of fixation. The differences in the results in Table 1 obtained from the near and far fixation distances clearly support this hypothesis. The results from the middle fixation condition (fixation on the point of light) on first consideration seem to be inconsistent with this hypothesis, since the average $D^{\prime}$ obtained from this condition is not intermediate between that from the near and far fixations. There are good reasons, however, not to use the middle fixation results to test this hypothesis. One reason is that the effect of the specific distance tendency is to make the point of light appear at about $200 \mathrm{~cm}$. This effect is in opposition to the effect on the apparent distance of the point of light of fixating at the near $(37 \mathrm{~cm})$ but not at the far $(168 \mathrm{~cm})$ distance. Thus, fixation at either the middle or far distance would be expected to produce similar values of $D^{\prime}$ with the head-motion procedure and, according to Table 1 , this occurred. Consistent with the effect of the specific distance tendency, the average value of $D^{\prime}$ of the point (with both procedures) was greater than its physical distance $(97.5 \mathrm{~cm})$, even when the fixation was at the near fixation distance.

With the size adjustment procedure, another factor is superposed upon the effect expected from the specific distance tendency. This second factor is the Aubert-Fleischl paradox (Aubert, 1887; Fleischl, 1882; Mack \& Herman, 1973), in which the perceived speed (and presumably the perceived extent of travel) of a moving object is less when the object is fixated than when the fixation is on a stationary point. For the size adjustment procedure with the fixation on the point of light, this paradox would increase the adjusted value of $S$ and thus would decrease $D^{\prime}$ (from Equation 1), as compared with the $\mathrm{D}^{\prime}$ obtained from conditions in which the fixation was on the stationary (near or far) fixation object. As shown in Table 1 , this effect is consistent with the results obtained using the size adjustment procedure with either the 3- or 5-in. criterion.

For both of these reasons (the presence of the specific distance tendency and the Aubert-Fleischl paradox), no measures of $D^{\prime}$ from the middle fixa- 
Table 1

Results in Centimeters from the Head-Motion and Size Adjustment Procedures

\begin{tabular}{|c|c|c|c|c|c|c|}
\hline & \multicolumn{2}{|c|}{ Fixation Near } & \multicolumn{2}{|c|}{ Fixation Middle } & \multicolumn{2}{|c|}{ Fixation Far } \\
\hline & $S$ & . $\mathrm{D}^{\prime}$ & $\mathbf{S}$ & $\mathrm{D}^{\prime}$ & $\mathbf{S}$ & $\mathbf{D}^{\prime}$ \\
\hline & \multicolumn{6}{|c|}{$\begin{array}{l}\text { Size Adjustment Procedure } \\
\qquad \mathrm{S}^{\prime}=7.6 \mathrm{~cm}(\mathrm{~N}=24)\end{array}$} \\
\hline $\begin{array}{l}\text { Mean } \\
\text { Geometric Mean } \\
\text { Median } \\
\text { SD }\end{array}$ & $\begin{array}{l}5.01 \\
4.07 \\
3.93 \\
3.52\end{array}$ & $\begin{array}{l}221 \\
182 \\
189 \\
141\end{array}$ & $\begin{array}{l}5.45 \\
4.87 \\
4.77 \\
2.86\end{array}$ & $\begin{array}{r}169 \\
153 \\
156 \\
77\end{array}$ & $\begin{array}{l}3.84 \\
3.06 \\
3.08 \\
3.02\end{array}$ & $\begin{array}{l}298 \\
243 \\
242 \\
203\end{array}$ \\
\hline SD & \multicolumn{6}{|c|}{$\mathrm{S}^{\prime}=12.7 \mathrm{~cm}(\mathrm{~N}=24)$} \\
\hline $\begin{array}{l}\text { Mean } \\
\text { Geometric Mean } \\
\text { Median } \\
\text { SD }\end{array}$ & $\begin{array}{l}9.62 \\
9.72 \\
8.76 \\
4.38\end{array}$ & $\begin{array}{r}156 \\
142 \\
141 \\
66\end{array}$ & $\begin{array}{l}9.26 \\
8.83 \\
9.00 \\
2.98\end{array}$ & $\begin{array}{r}147 \\
140 \\
138 \\
43\end{array}$ & $\begin{array}{l}7.33 \\
6.61 \\
6.59 \\
3.41\end{array}$ & $\begin{array}{r}207 \\
187 \\
188 \\
95\end{array}$ \\
\hline \multirow{2}{*}{ SD } & \multicolumn{6}{|c|}{$\begin{array}{l}\text { Head-Motion Procedure } \\
\qquad(\mathrm{N}=48)\end{array}$} \\
\hline & W & $\mathrm{D}^{\prime}$ & W & $\mathrm{D}^{\prime}$ & W & $\mathrm{D}^{\prime}$ \\
\hline $\begin{array}{l}\text { Mean } \\
\text { Geometric Mean } \\
\text { Median } \\
\text { SD }\end{array}$ & $\begin{array}{l}1.51 \\
- \\
2.42 \\
5.10\end{array}$ & $\begin{array}{r}117 \\
112 \\
113 \\
34 \\
\end{array}$ & $\begin{array}{l}7.12 \\
7.08 \\
6.99 \\
0.76 \\
\end{array}$ & $\begin{array}{r}165 \\
165 \\
162 \\
12 \\
\end{array}$ & $\begin{array}{l}7.08 \\
7.04 \\
7.13 \\
0.70\end{array}$ & $\begin{array}{r}164 \\
164 \\
165 \\
11 \\
\end{array}$ \\
\hline
\end{tabular}

Note-With the size adjustment procedure, $S$ is the physical horizontal motion required in order for the point of light physically moving horizontally to appear to move $\left(S^{\prime}\right)$ either $3 \mathrm{in} .(7.6 \mathrm{~cm})$ or $5 \mathrm{in} .(12.7 \mathrm{~cm})$ and $D^{\prime}$ is the perceived distance of the point of light found from $S$ and $S^{\prime}$ using Equation 1 . With the head-motion procedure, $W$ is the physical horizontal motion required for the point of light physically moving with a vertical component to appear to move vertically and $D^{\prime}$ is the perceived distance of the point of light found from $W$ using Equation 2.

tion condition were used in testing the main hypothesis of this study. The $\mathrm{D}^{\prime}$ results obtained using the head-motion procedure and fixation on the point of light is of interest, however, in that they indicate that errors can occur in the perceived distance of an object (as a result of the specific distance tendency), even though the object is fixated. Casual observation by the experimenter suggests that, as indicated by the presence of apparent concomitant motion, errors in the perceived distance of fixated objects also are not uncommon in more normal visual fields.

The $\mathrm{D}^{\prime}$ of the point of light obtained from the far fixation distance was greater than that obtained from the near fixation distance for 40 out of 48 of the observers with the size adjustment procedure and for 46 out of 48 of the observers with the headmotion procedure. According to the test, $D^{\prime}$ increased significantly as the fixation was changed from near to far as measured by the size adjustment procedure for $S^{\prime}=3$ in. $[\mathrm{t}(23)=2.66, \mathrm{p}<.01]$ and for $\mathrm{S}^{\prime}=5$ in. $[\mathrm{t}(23)=3.98, \mathrm{p}<.01]$ and also as measured by the head-motion procedure [t(47) $=9.86, \mathrm{p}<.01]$.

The verbal reports of the apparent distance of the point of light are given in Table 2 . Since the distributions of verbal reports are often skewed, the median reports are considered to best represent the data. Although the median reported distance of the point of light was greater with the far than with the near fixation (as obtained following either the size adjustment or head-motion procedure), these differences were not significant at the .05 level as tested by the Wilcoxin sign test for matched pairs, $Z=1.35$ and .99 , respectively. According to the verbal reports of the apparent distance of the fixation objects (verbal reports not shown in Table 2), the far fixation object appeared at a greater distance than the near fixation object for 47 out of 48 observers with median reports of $30.5 \mathrm{~cm}$ for the near and $91.4 \mathrm{~cm}$ for the far fixation object. Also, the average size adjusted between the visible bars in the observation booth to equal 3 and 5 in. was fairly accurate, i.e., 3.78 and 4.96 in., respectively.

\section{DISCUSSION}

Although both the head-motion and size adjustment procedures provide strong support for the effect of fixation distance upon the perceived distance of nonfixated objects, the results from the two procedures differ clearly in several respects. The most obvious difference is that the measurement of $\mathrm{D}^{\prime}$ is considerably larger from the size adjustment procedure than from the head-motion procedure, except when the fixation is on the point of light. The similarity of the measured $D^{\prime}$ from the two procedures with the fixation on the point of light suggests that it is the larger $D^{\prime}$ (smaller $S$ ) results from the 
Table 2

Verbal Reports of the Apparent Distance of the Point of Light Following the Use of Either the Size Adjustment or Head-Motion Procedure

\begin{tabular}{|c|c|c|c|c|c|c|}
\hline & \multicolumn{3}{|c|}{ Following Size Adjustment } & \multicolumn{3}{|c|}{ Following Head Motion } \\
\hline & $\begin{array}{c}\text { Fixation } \\
\text { Near }\end{array}$ & $\begin{array}{c}\text { Fixation } \\
\text { Middle }\end{array}$ & $\begin{array}{c}\text { Fixation } \\
\text { Far }\end{array}$ & $\begin{array}{c}\text { Fixation } \\
\text { Near }\end{array}$ & $\begin{array}{c}\text { Fixation } \\
\text { Middle }\end{array}$ & $\begin{array}{c}\text { Fixation } \\
\text { Far }\end{array}$ \\
\hline Mean & 158 & 105 & 152 & 129 & 113 & 151 \\
\hline Geometric Mean & 106 & 92 & 118 & 92 & 96 & 116 \\
\hline Median & 91 & 102 & 122 & 107 & 91 & 122 \\
\hline SD & 226 & 53 & 134 & 121 & 75 & 160 \\
\hline
\end{tabular}

size adjustments at the near and far fixation that require explanation. It seems that the perceived extent of lateral motion of the point of light was overestimated when the fixation was not on the point of light. This interpretation, although consistent with the Aubert-Fleischl paradox, is not consistent with the interpretation of this paradox in terms of the underestimation of pursuit eye movements associated with fixation on the moving point (Mack \& Herman, 1973). A discrepancy in the size adjustment data, for which the author has no ready explanation, is the larger average $\mathrm{D}^{\prime}$ obtained from the 3 -in. $(7.6-\mathrm{cm})$ than from the 5 -in. $(12.7-\mathrm{cm})$ criterion.

It is of interest to compare the interobserver variability of the $D^{\prime}$ of the point as obtained from the head-motion procedure, the size adjustment procedure, and the verbal reports. For the same fixation distance, the head-motion procedure clearly provides the least variable measure of $D^{\prime}$ with the other two procedures about equal in variability.

\section{Theoretical Implications}

Implications for the theory of direct perception. Gibson's theory of direct perception is "primarily a theory of correct perception" (Gibson, 1966, p. 287). Only certain kinds of errors in perception, however, provide a challenge to this theory. First, the errors must not be due simply to a lack of precision, i.e., they must be mean or constant errors, not variable errors. For instance, the precision and consistency of perception in the human infant is likely to increase as an infant gets older, but this presents no theoretical problem to the theory of direct perception. Also, only certain kinds of mean errors need to be considered. These must be constant errors not attributable to distortions in the optic array, such as might be produced by optical devices. The constant errors in perception that would trouble this theory are those that occur because of the manner in which valid information in the optic array is processed within the observer. In the present study, the errors in the perception of the point of light as a function of fixation distance clearly are errors of this latter type. Finally, the challenge generated by such constant errors is increased if they occur under normal conditions of observation. The present study which used monocular observation and a point of light suspended in a visual alley (albeit a well-structured visual alley), does not constitute normal or usual conditions of observation. The significance of this study, together with that by Gogel and Tietz (1977), however, is that it provides strong support for the notion that the apparent motion concomitant with head motion that frequently occurs in everyday environments is a consequence of errors in perceived distance. This notion is seriously inconsistent with the theory of direct perception.

Rock $(1975,1977)$ suggests that visual perception, in addition to its more distally oriented qualities, has an aspect that is closely related to the retinal characteristics of the stimulus. In size and shape perception, this second aspect is an impression corresponding to visual angle and retinal shape, respectively. In the perception of position, it is an impression of movement (pseudomovement) despite the more salient perception of a stationary object. A similar distinction, in the case of motion perception, has been discussed by Mack (1978). These two aspects, or modes, of perception are called the proximal and constancy modes and are similar to the earlier distinction made by Gibson (1950) between the visual field and visual world. More recently, Gibson (1966), in discussing the finding that constancy is often not complete, suggests that sensations (as distinct from perceptions) can sometimes intrude into the judgments of the visual world. Similarly, Rock (1977) indicates that the two modes of perception may be involved in a variety of perceptions involving some degree of underconstancy.

Can the illusory motions of stationary objects concomitant with the motion of the head be considered in this manner? If so, perhaps such pseudomotions should not be interpreted as indicating an error in perceived distance. There are a number of reasons for rejecting this interpretation. One reason is that in the present experiment the measurement of apparent distance using illusory concomitant motions as a function of fixation is in general agreement with the results obtained from the size adjustment procedure. Second, the results from the head-motion procedure using the illusory concomitant motion are consistent with the effect of fixation upon apparent distance 
found by Wist and Summons (1976). Third, there is a compelling reason for not considering the illusory concomitant motion as a pseudomotion different from the usual perception of motion. It will be recalled that the head-motion procedure used a physical component of concomitant vertical motion which produced a perceptual component of motion $\left(V^{\prime}\right)$. This component, $V^{\prime}$, vectored with the component of illusory concomitant motion $\left(\mathrm{W}^{\prime}\right)$ to produce the perception of the path of motion of the point of light. It seems reasonable that if $\mathrm{V}^{\prime}$ and $\mathrm{W}^{\prime}$ can be added vectorially, they must be processed by the visual system as phenomena of the same kind. Conversely, the present study indicates that the distinction between perceptions and sensations indicated by Gibson's analysis, or between proximal and constancy modes of perception, is unnecessary and probably ought to be discarded. It should be noted that if this conclusion is valid, a theory of perception such as Gibson's theory cannot use a distinction between sensation and perception to limit the subjective phenomena that it is required to explain.

The postulation of a proximal mode of perception is perilously close to the notion that, under some conditions, the observer can respond directly to (perceive) the retinal image. Figure 5 illustrates conditions that explore further the inconsistency between the implications of the present study and the explanation of the illusory concomitant motion as a direct response to motion on the eye. The situation is shown in Figure 5 in which two points of light physically at a and $b$ but perceptually at $a^{\prime}$ and $b^{\prime}$ are viewed as the head is moved between Positions 1 and 2. In Figure 5A, the perceived depth between Points $a$ and $b$ is less than the physical depth. As a result of this error in perceived depth, Point a appears to move against the head relative to Point $b$. This is consistent with the relative motions of the images of Points a and $b$ on the eye. In Figure 5B, however, the relative motions of the images of Points $a$ and $b$ on the eye are inconsistent with their perceived relative motions. The difference between Figures 5A and 5B is that, in Figure 5B, the perceived depth between the points is greater (not less) than the physical depth. It follows that, depending upon the direction of the error in perceived depth, apparent concomitant motion can

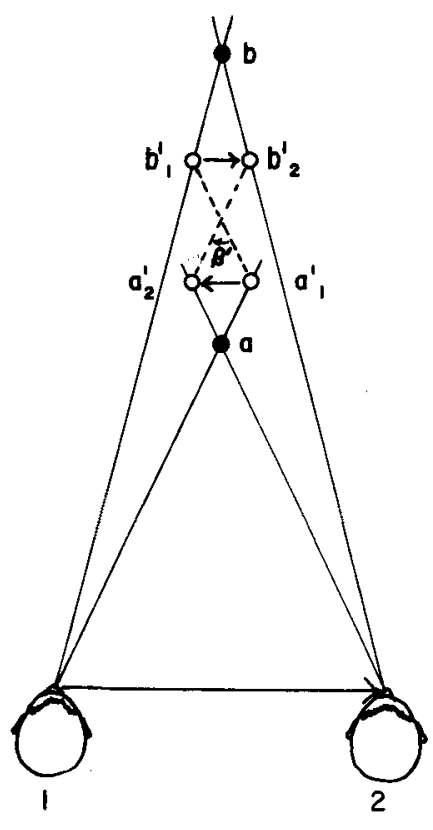

A

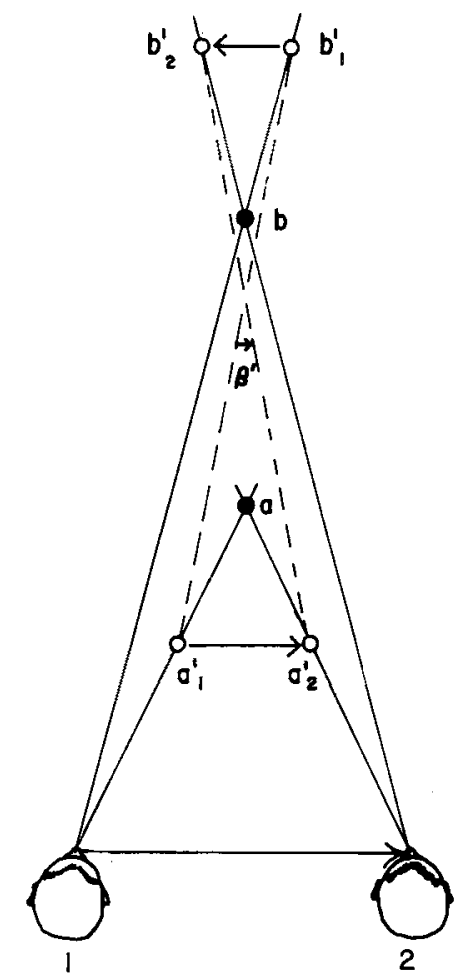

B

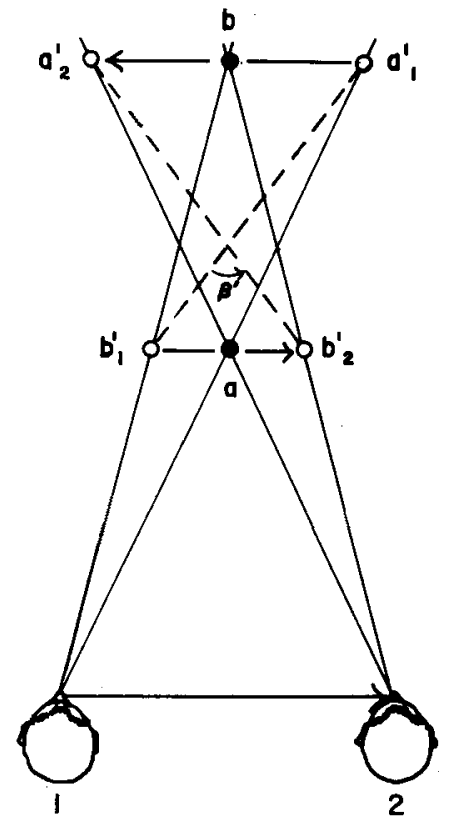

C

Figure 5. Perceived concomitant rotation, $\beta$; of Points a and $b$ physically at different distances from the observer as the head is moved horizontally. In $A$, the perceived depth is less than the physical depth and the perceived rotation is consistent in direction with that expected from the relative motions on the eye. In $B$, the perceived depth is greater than the physical depth and it is expected from the geometrical considerations that the direction of perceived rotation will be inconsistent with the retinal motions. In C, a perception of rotation superficially similar to B occurs, but, in this case, the direction of apparent rotation is a consequence of Point $b$ being perceptually closer but physically more distant than Point a. This last case is illustrated by a physically concave, but perceptually convex, mask of a face that appears to turn in the direction of the observer's motion. 
be in the same direction or opposite to the direction expected from the relative motion on the eye. That apparent concomitant motion is almost always consistent with the retinal motion is a happenstance of the prevalence of the perceptual underestimation rather than overestimation of the depth between objects, with one important source of this underestimation attributable to the tendency for objects to appear near the distance of fixation. This can be considered to be a special case (see Gogel \& Tietz, 1977) of the tendency for objects to appear at the same distance (the equidistance tendency). The high rate of occurrence of the apparent concomitant motions illustrated in Figure 5A and the low rate of those illustrated in Figure $5 \mathrm{~B}$ attests to the prevalence of equidistance effects in the perception of depth. Since there seems to be no tendency to perceive more depth than that found physically, the situation of Figure 5B is apt to occur under only very contrived laboratory conditions. That the perception predicted for Figure 5B will actually occur, however, remains to be demonstrated.

Implications for the perception of rotation. If Points $a$ and $b$ in Figure 5 are regarded as the ends of an extended object ab shown by the dashed lines, Figure 5 can also be used to explain an apparent rotation of objects or scenes that occurs as a consequence of a misperception of depth. In Figure 5A, as the observer moves between Positions 1 and 2, Object $\mathrm{ab}$ appears to rotate with a magnitude and direction indicated by $\beta^{\prime}$. In Figure 5B, Object ab appears to rotate opposite to the apparent rotation indicated in Figure 5A with the rotation in the latter direction increased in the case of Figure 5C. An instance of Figure 5C described by Gregory (1970) is a physically concave mask of a human face that appears convex and also appears to turn in the direction of the moving observer. As is illustrated in Figure 5 (also, see Gogel \& Tietz, 1974), the relation between errors in depth perception and apparent concomitant motion can be applied to a variety of physically stationary scenes that appear to a moving observer to rotate.

Other perceptual phenomena associated with errors in perceived distance. Errors in perceived distance can produce a variety of perceptual effects concomitant with different kinds of observer and object motion. Gregory and Ross (1964a, 1964b) have shown that, under reduced conditions of observation, observer motion toward or away from an object can result in a change in perceived object size and often the object will appear to move in distance. Binocular observation and proprioceptive information regarding the observer's motion decrease, but do not eliminate, these effects. Conversely, it is likely that these changes in apparent object size and location as the observer moves toward or away from objects can be used to measure errors in the apparent distances of the objects. That some of these effects also can occur with a stationary observer and moving object is shown, for example, with the concave mask that appears convex. Moving or tilting the mask will produce changes in its apparent orientation similar or identical to those from optically equal motion of the observer's head.

There are other instances of apparent motion concomitant with motion of the head that result from misperceptions of distance. A trapezoidal window with its small (right) end physically closer to the observer than its large (left) end will appear oppositely oriented in depth. Moving the head right and left will result in an apparent depth rotation of the window generally consistent with that illustrated in Figure 5C. Also, moving the head up and down will result in an apparent clockwise and counterclockwise rotation of the window. In addition, the perception of motion of near portions of the visual field relative to far portions is readily noted by a rapidly moving observer, for example, during a train ride (Rock, 1975, p. 115). It is the point of view of this study that this perception of motion perspective is directly the result of observer errors in the perception of depth within the physically stationary scene. If motion perspective or motion parallax is effective as a distance cue, its effect is to produce a correct perception of distance in a physically stationary scene, not a perception of relative motion. The perception of concomitant motion in scenes having motion perspective, to the extent that this occurs, is an indication of errors in the perception of depth and, thus, is an indication of some lack of effectiveness of motion perspective as a cue to distance. Rock (1977) has noted that directional adjacency, an analytic attitude, and large amounts of depth or distance will contribute to perception in the proximal mode. In support of the viewpoint of the present study, these same factors are also likely to contribute to the underperception of the depth between objects.

Implications for the perception of direction. The small variability in the measurement of perceived distance from the head-motion procedure indicates that the perception of direction to an object viewed with moving head is quite precise. It is as though a definite and rather precise geometry of perceived direction that matches the physical world were available to the observer even though perceived distance and perceived motion are in error. This is consistent with a study by Foley (1975), using a stationary head in which it was found that the perception of direction, unlike the perception of distance, was uninfluenced by whether the conditions of observation were multicue or more reduced.

The head-motion procedure is based upon the existence within the visual system of a stable relation between perceived concomitant motion ( $\left.\mathrm{W}^{\prime}\right)$, perceived change in direction $\left(\phi^{\prime}\right)$ per unit of sensed head 
motion $\left(\mathrm{K}^{\prime}\right)$, and perceived distance $\left(\mathrm{D}^{\prime}\right)$. The relation between these variables (Gogel \& Tietz, 1974) is

$$
\mathbf{W}^{\prime}=\mathbf{K}^{\prime}-\phi^{\prime} \mathbf{D}^{\prime} \text {. }
$$

Equation 3 is a perceptual equation to be distinguished from a psychophysical equation that relates only a single perceptual dimension to physical stimuli (Gogel, 1973). The size-distance invariance hypothesis also expresses a perceptual equation which, in terms of perceptual and proximal variables, is that

$$
\mathrm{D}^{\prime}=\mathrm{CS}^{\prime} / \theta
$$

where $\theta$ in radians equals $S / D$ in Equation 1 . As is indicated in the present study by means of perceptual equations, one perception can be used to measure another. Also, as shown, the use of different perceptual equations involving a common perceptual variable provides additional evidence for the validity of the procedures used in obtaining the measure of the perception common to the two equations.

\section{Summary}

This study adds to the evidence that the visual world of the observer is geometrically organized and that this organization can be expressed by equations (such as Equations 3 and 4) that specify the interrelations of different perceptions. It asserts that errors in perceived distance are reflected in a variety of perceptual phenomena. The ubiquitous occurrence of these phenomena, particularly the phenomenon of illusory motion concomitant with the motion of the head, indicates, contrary to the theory of direct perception, that constant errors in perceived distance are frequent even in well-structured visual fields. Furthermore, the study provides experimental evidence in support of Boring's contention (Boring, 1952) that the distinction between the visual world and visual field made by Gibson (1950) and the more recent revivals of this distinction in terms of perceptual modes (Mack, 1978; Rock, 1977) is unnecessary.

\section{REFERENCES}

Aubert, H. Die Bewegungsempfindungen. Pflügers Archiv ges. Physiol., 1887, 40, 459-480.
Boring, E. G. Visual perception as invariance. Psychological Review, 1952, 59, 141-148.

FleisChl, E. Physiologisch-optische Notizen. Sitzungsberichte der Akademie der Wissenschaften, 1882, 89, 17-25.

Foley, J. M. Error in visually directed manual pointing. Perception \& Psychophysics, 1975, 17, 69-74.

GiBson. J. J. The perception of the visual world. Boston: Hougton Mifflin, 1950.

GiBson, J. J. The senses considered as perceptual systems. Boston: Houghton Mifflin, 1966.

Goged, W. C. The organization of perceived space. II. Consequences of perceptual interactions. Psychologisch Forschung, 1973. 36, 223-247.

GoGel, W. C. An indirect method of measuring perceived distance from familiar size. Perception \& Psychophysics, 1976, 20, 419-429.

GoGEL. W. C. An indirect measure of perceived distance from oculomotor cues. Perception \& Psychophysics, 1977, 21, 3-11.

Gogel, W. C., \& Tietz, J. D. Absolute motion parallax and the specific distance tendency. Perception \& Psychophysics, 1973, 13, 284-302.

Gogel, W. C., \& Tietz, J. D. The effect of perceived distance on perceived movement. Perception \& Psychophysics, 1974, 16, $70-78$.

Gogel, W. C., \& TIETz, J. D. Eye fixation and attention as moditiers of perceived distance. Perceptual and Motor Skills, 1977, 45, 343-362.

GREGORY, R. L. The intelligent eye. New York: McGraw-Hill, 1970.

GrEGORY, R. L., \& Ross, H. E. Visual constancy during movement. 1. Effects of S's forward and backward movement on size constancy. Perceptual \& Motor Skills, 1964, 18, 3-8. (a)

GREGORY, R. L., \& Ross, H. E. Visual constancy during movement. 2. Size constancy using one eye or both eyes or proprioceptive information. Perceptual \& Motor Skills, 1964, 18, 23-26. (b)

Kilpatrick, F. P., \& Ittelson, W. H. The size-distance invariance hypothesis. Psychological Review, 1953, 60, 223-231.

MACK. A. Three modes of visual perception. In H. L. Pick \& E. Saltzman (Eds.), Modes of perceiving and processing information. New York: Wiley, 1978.

Mack, A., \& Herman, E. Position constancy during pursuit eye movement: An Investigation of the Filehne illusion. Quarterly Journal of Experimental Psychology, 1973, 25, 71-84.

Rock, I. An introduction to perception. New York: Macmillan, 1975.

Rock, I. In defense of unconscious inference. In W. Epstein (Ed.), Stability and constancy in visual perception. New York: Wiley, 1977.

Wist, E. R., \& Summons, E. Spatial and fixation conditions affecting the temporal course of changes in perceived relative distance. Psychological Research, 1976, 39, 99-112.
(Received for publication May 15, 1978; revision accepted October 27,1978 .) 